\title{
Primary Focal Segmental Glomerulosclerosis
}

National Cancer Institute

\section{Source}

National Cancer Institute. Primary Focal Segmental Glomerulosclerosis. NCI Thesaurus. Code C45434.

Focal scarring of the glomerulus, which may result in isolated proteinuria or nephrotic syndrome, which affects only part of the glomerulus and only some of the glomeruli. The condition is considered primary in cases for which no underlying cause has been identified. 